\title{
A Presença de Retocele Interfere nos Resultados de Exames de Fisiologia Anal?
}

\author{
Rectocele Influences Results of Anal Physiological Examinations?
}

\author{
MARIA AUXILIADORA PROLUNGATTI CESAR ${ }^{1}$; WILMAR ARTUR KLUG ${ }^{2}$ HELLY ANGELA CARAM AGUIDA ${ }^{3}$; \\ JORGE ALBERTO ORTIZ ${ }^{4}$; FANG CHIA BIN ${ }^{5}$; PERETZ KAPELHUCHNIK ${ }^{6}$
}

\begin{abstract}
${ }^{1}$ Mestre e Doutora em Cirurgia - Área de Coloproctologia da Faculdade de Ciências Médicas da Santa Casa de São Paulo. Professor Assistente Doutor da Universidade de Taubaté; ${ }^{2}$ Professor Titular Livre Docente da Faculdade de Ciências Médicas da Santa Casa de São Paulo. Chefe da Área de Coloproctologia; ${ }^{3}$ Mestre em Cirurgia - Área de Coloproctologia - Pela Faculdade de Ciências Médicas da Santa Casa de São Paulo; ${ }^{4}$ Mestre em Cirurgia - Área de Coloproctologia - Pela Faculdade de Ciências Médicas da Santa Casa de São Paulo. Responsável pelo Ambulatório de Fisiologia Anal do Departamento de Cirurgia - Área de Coloproctologia da Faculdade de Ciências Médicas da Santa Casa de São Paulo; ${ }^{5}$ Mestre e Doutor em Cirurgia da Faculdade de Ciências Médicas da Santa Casa de São Paulo; ${ }^{6}$ Professor Titular Livre Docente da Faculdade de Ciências Médicas da Santa Casa de São Paulo - Brasil.
\end{abstract}

\begin{abstract}
CESAR MAP; KLUG WA; AGUIDA HAC; ORTIZ JA; BIN FC; KAPELHUCHNIK P. A Presença de Retocele Interfere nos Resultados de Exames de Fisiologia Anal? Rev bras Coloproct, 2008;28(3): 329-333

RESUMO: INTRODUÇÃO - A retocele é causa comum de constipação por defecação obstruída. Freqüentemente está relacionada com outras causas de defecação obstruída, e os exames de fisiologia anal são importantes para o diagnóstico preciso da causa de constipação intestinal. OBJETIVO - observar a influência da retocele nos exames de fisiologia anal, e a necessidade da realização desses após o diagnóstico da retocele. MÉTODO - Para este trabalho foram estudados 40 pacientes com diagnóstico de defecação obstruída. Todos os pacientes foram submetidos aos exames de manometria, sensibilidade retal, eletromiografia, latência do nervo pudendo e proctografia, e foram separados em 2 grupos: portadores e não portadores de retocele . Foram encontrados 18 pacientes com retocele, e o diagnóstico mais freqüentemente associado foi o anismus . Em relação aos exames foi encontrada diferença significante apenas na proctografia no ângulo ano retal, demonstrando que a presença de retocele não interferiu nos resultados de exames de fisiologia anal quando comparados com outros constipados, mas esteve associada a outras causas de constipação, como anismus que necessita de outro tipo de tratamento. CONCLUSÃO - A retocele não interferiu nos valores dos exames de fisiologia anal, mas esteve associada a outros diagnósticos, sendo importante a realização dos mesmos.
\end{abstract}

Descritores: Retocele; Constipação Intestinal; Técnicas de Diagnóstico e Procedimentos.

\section{INTRODUÇÃO}

A constipação intestinal pode ser definida como a ocorrência de menos que três evacuações por semana ou evacuação com dificuldade, incompleta ou prolongada $(13 ; 12 ; 11)$ podendo se classificar em 2 tipos principais : transito colônico lento ou inércia colônica e defecação obstruída $(2 ; 3 ; 7 ; 6)$.

Dentre as causas da defecação obstruída a retocele é uma bolsa da parede anterior do reto e da parede vaginal posterior para a luz da vagi- na, e que freqüentemente é encontrada quando as pacientes são investigadas por constipação (8), considerada clinicamente quando maior que 20 mm. (5)

É achado comum e pode ser considerada uma variável normal, e sua contribuição na desordem evacuatória é complexa e não clara (10;4).

São sintomas da retocele o esvaziamento incompleto, sensação de pressão retal e sintomas vaginais. Pacientes constipados usualmente tem sintomas similares $(8,9)$. 
O diagnóstico se faz através do exame físico e/ou da proctografia, sendo que o seu tamanho é calculado durante o esforço como a distância do ápice da parede anterior do reto para uma linha no eixo do canal anal. É encontrada em $25 \%$ das proctografias de constipados $(7,8,9)$.

O objetivo deste trabalho é rever os pacientes cujo diagnóstico no ambulatório de fisiologia anal foi retocele, e observar as variações dos exames de fisiologia anal verificando a importância da realização dos mesmos.

\section{CASUÍSTICA E MÉTODO}

O trabalho realizado consistiu no estudo de fichas de 40 pacientes com constipação intestinal, provenientes do ambulatório de Fisiologia anal da área de coloproctologia da Santa Casa de Misericórdia de São Paulo, e que realizaram os exames completos de fisiologia anal pela autora.

\section{Manometria Ano-Retal}

Utilizado o aparelho Proctosystem PL-3000, manômetro de balão. Os exames foram realizados sem preparo intestinal, colocando-se o paciente em decúbito lateral esquerdo.

Foram consideradas a maior pressão de repouso obtida, a maior pressão de contração e a menor pressão de evacuação no canal anal. No reto foi considerada a máxima pressão retal de repouso e a máxima pressão retal de evacuação.

\section{Sensibilidade Retal}

Avaliada através da utilização de um balão confeccionado com preservativo, com forma alongada, de aproximadamente seis centímetros de comprimento conectado à uma sonda retal, e esta à uma seringa de $50 \mathrm{ml}$.

a) Menor volume sensível: era o limiar, o primeiro volume que provocava o desejo de evacuar ou a primeira sensação de peso retal que desaparecia após 30 segundos.

b) Volume de Sensação Constante: o ar insuflado promovia um desejo de evacuar que era permanente.

c) Volume máximo tolerável: era o volume máximo que promovia um desejo incontrolável de evacuar. d) Evacuação do balão: o paciente em decúbito lateral esquerdo era solicitado a realizar o esforço evacuatório para evacuar o balão. Não era ajudado inicialmente pelo examinador.

\section{Proctografia}

Introdução de $120 \mathrm{ml}$ de contraste baritado na ampola retal através de sonda retal, com o paciente em decúbito lateral esquerdo. Após a introdução do contraste eram realizadas três radiografias na posição lateral em repouso, contração e evacuação.

$\mathrm{Na}$ avaliação das proctografias foram traçadas as seguintes linhas nas três radiografias:

a) Linha pubo-coccígea: linha traçada entre a parte superior da pube e o cóccix;

b) Canal anal: linha que se inicia no orifício anal e se estende até a junção ano-retal;

c) Eixo retal: linha marcada no eixo longitudinal do reto em sua parede posterior.

Após traçadas as linhas foram realizadas as seguintes medidas:

a) Posição de diafragma pélvico: medida entre a extremidade superior do canal anal e a linha pubococcígea através de uma linha perpendicular;

b) Posição perineal: medida entre a extremidade inferior do canal anal e a linha pubo-coccígea através de uma linha perpendicular;

c) Medida do canal anal;

d) Ângulo ano-retal: medido entre o eixo do reto e do canal anal no ponto de interseção das linhas.

\section{Período de Latência do Nervo Pudendo}

Realizado através da introdução de luva apropriada com eletrodo na ponta do dedo indicador e em sua base. Eram dados estímulos ao nível da espinha isquiática nas proximidades do nervo pudendo, aferindo-se o tempo entre o estímulo no nervo pudendo e a resposta ao nível do ânus. Os valores considerados normais estavam no intervalo de 1,8 a $2,3 \mathrm{~ms}$.

Dos 40 pacientes 18 eram portadores de retocele e 22 não portadores, sendo os dois grupos comparados em relação aos resultados dos exames de fisiologia anal.

A análise estatística se fez através do teste $\mathrm{t}$ de Student para comparação de médias, controlado pelo teste de Levene para igualdade das variâncias. 


\section{RESULTADOS}

Foram estudados 40 pacientes com queixas de constipação intestinal por defecação obstruída, sendo todos submetidos à avaliação completa através dos métodos disponíveis no laboratório de fisiologia anal.

A retocele esteve presente em 18 dos 40 pacientes constipados (45\%). Em quatro dos 18 pacientes portadores de retocele $(22,22 \%)$ esta foi a única causa de constipação. A contração paradoxal do pubo-retal foi a causa de defecação obstruída mais freqüentemente associada à retocele, e ocorreu em 8 dos 18 pacientes (44,44\%), a intussuscepção ocorreu em cinco dos 18 $(27,77 \%)$, e a sigmoidocele em quatro dos $18(22,22 \%)$.

Em dez dos 18 pacientes $(55,56 \%)$ houve uma causa associada, em três dos 18 pacientes $(16,66 \%) 2$ causas associadas, em um dos 18 pacientes $(5,56 \%) 3$ causas associadas, e foi a causa única em quatro dos 18 pacientes $(22,22 \%)$.
Os pacientes foram divididos em dois grupos: 18 eram portadores de retocele e os demais (22 pacientes) não apresentavam esta afecção.

Os dois grupos não apresentaram diferenças estatisticamente significantes em relação à ritmo intestinal com a utilização de laxantes, ritmo intestinal sem a utilização de laxantes, duração da queixa, paridade e idade.

Através da manometria foram observadas que as médias das pressões de repouso, contração e evacuação não apresentaram diferença estatisticamente significante (Tabela 1).

A avaliação da sensibilidade retal em pacientes portadores de retocele, comparados com os sem retocele, não mostram diferenças significantes, embora o volume máximo tolerável fosse maior no grupo com retocele (Tabela 2).

A latência do nervo pudendo também não demonstrou diferenças estatisticamente significantes entre os grupos estudados (Tabela 3).

Tabela 1 - Médias de pressões anais e retais em constipados portadores ou não de retocele.

\begin{tabular}{lccc}
\hline Manometria & Retocele & Não portadores de retocele & p \\
\hline Máxima pressão de repouso & $66,61 \pm 17,84$ & $57,23 \pm 18,19$ & $\mathrm{Ns}$ \\
Máxima pressão de contração & $142,94 \pm 63,20$ & $124,41 \pm 42,31$ & $\mathrm{Ns}$ \\
Mínima pressão de evacuação & $34,78 \pm 13,53$ & $33,41 \pm 11,69$ & $\mathrm{Ns}$ \\
Pressão retal & $11,83 \pm 16,14$ & $14,59 \pm 8,83$ & $\mathrm{Ns}$ \\
Pressão retal de evacuação & $62,5 \pm 25,54$ & $52,09 \pm 17,46$ & $\mathrm{Ns}$ \\
Zona de hiperpressão & $3,06 \pm 0,94$ & $3,36 \pm 0,73$ & $\mathrm{Ns}$ \\
\hline
\end{tabular}

Tabela 2 - Médias de sensibilidade retal em constipados portadores ou não de retocele.

\begin{tabular}{lccc}
\hline Sensibilidade retal & Retocele & Não portadores de retocele & p \\
\hline Volume que provoca o primeiro desejo de evacuar & $60 \pm 22,16$ & $71,23 \pm 31,90$ & Ns \\
Volume de sensação constante & $121,11 \pm 41,18$ & $135 \pm 42,48$ & Ns \\
Volume máximo tolerável & $334,44 \pm 158,16$ & $280,45 \pm 61,37$ & Ns \\
\hline
\end{tabular}

Tabela 3 - Médias de latência do nervo pudendo em constipados portadores ou não de retocele.

\begin{tabular}{lccc}
\hline Latência do nervo pudendo & Portadores de retocele & Não portadores de retocele & p \\
\hline Direita & $2,28 \pm 0,75$ & $2,05 \pm 0,49$ & $\mathrm{Ns}$ \\
Esquerda & $2,17 \pm 0,71$ & $2,27 \pm 0,88$ & $\mathrm{Ns}$ \\
\hline
\end{tabular}


Observando as proctografias de portadores de retocele e não portadores de retocele, pudemos verificar que existiram diferenças estatisticamente significantes entre constipados com e sem retocele em relação ao ângulo ano-retal na contração, posição de diafragma pélvico e posição perineal na evacuação (Tabela 4).

\section{DISCUSSÃO}

A retocele é causa importante da constipação intestinal por defecação obstruída. Os critérios diagnósticos variam, há os que consideram os aspectos clínicos, e alguns autores quando é achado exclusivo da proctografia e não de exame físico e história. Esses autores consideram a retocele, e a esta indicam tratamento cirúrgico de acordo com história, exame físico e tamanho maior que dois centímetros na proctografia $(7,8,9)$.

Em vista dos resultados obtidos através da proctografia pudemos verificar que, além da história e do exame físico, este é o método mais importante no diagnóstico de retocele. A mesma foi encontrada em 18 dos 40 (45\%)pacientes, sendo que foi diagnóstico isolado em quatro dos 18 pacientes $(22,22 \%)$, associado a contração paradoxal do pubo-retal em oito dos 18 pacientes $(44,44 \%)$.

Quando estudamos os resultados em pacientes com e sem retocele através da manometria, sensibilidade retal, eletromiografia, latência do nervo pudendo e proctografia não observamos diferenças estatisticamente significantes, como podemos observar na Tabs. $1,2,3$ e 4 .

Como pudemos observar no estudo da eletromiografia, $36,84 \%$ dos portadores de retocele apresentaram aumento da atividade mioelétrica no esforço evacuatório, refletindo algum grau de contração paradoxal do músculo pubo-retal. Esse dado é importante, pois é mecanismo associado na gênese da constipação, e pode necessitar de outro tipo de abordagem terapêutica.

Apesar de não ocorrer diferença estatisticamente significante entre os valores dos exames de fisiologia anal, a importância de se estudar a influência da retocele nos exames existe, pois como pudemos observar encontramos outros diagnósticos associados e a presença desses pode levar a um resultado ruim no tratamento, como por exemplo, quando existe a contração paradoxal do puborretal, que deve ser tratada antes da correção cirúrgica da retocele.

\section{CONCLUSÃO}

A retocele não interferiu nos valores dos exames de fisiologia anal, mas esteve associada a outras causas de constipação demonstradas através dos exames, sendo importante a realização dos mesmos mesmo com o diagnóstico prévio de retocele.

Tabela 4 - Médidas na proctografia em constipados portadores ou não de retocele.

\begin{tabular}{lrrc}
\hline Medidas de proctografia & Com retocele & Sem retocele & p \\
\hline Ângulo ano-retal no repouso & $102,44 \pm 12,51$ & $108,18 \pm 17,98$ & $\mathrm{Ns}$ \\
Comprimento do canal anal no repouso & $3,16 \pm 1,43$ & $3,76 \pm 1,15$ & $\mathrm{Ns}$ \\
Posição do diafragma pélvico no repouso & $6,56 \pm 2,27$ & $6,06 \pm 2,29$ & $\mathrm{Ns}$ \\
Posição perineal no repouso & $9,19 \pm 2,30$ & $8,60 \pm 2,00$ & $\mathrm{Ns}$ \\
Ângulo ano-retal na contração & $79,83 \pm 11,24$ & $88,82 \pm 13,71$ & $\mathrm{P}<0,05$ \\
Comprimento do canal anal na contração & $6,63 \pm 6,48$ & $6,25 \pm 2,52$ & $\mathrm{Ns}$ \\
Posição do diafragma pélvico na contração & $4,36 \pm 1,59$ & $3,94 \pm 1,65$ & $\mathrm{Ns}$ \\
Posição perineal na contração & $7,82 \pm 1,30$ & $7,16 \pm 1,63$ & $\mathrm{Ns}$ \\
Ângulo ano-retal na evacuação & $111,17 \pm 12,88$ & $117,86 \pm 11,42$ & $\mathrm{Ns}$ \\
Comprimento do canal anal na evacuação & $1,88 \pm 0,99$ & $2,64 \pm 1,34$ & $\mathrm{Ns}$ \\
Posição do diafragma pélvico na evacuação & $8,08 \pm 2,16$ & $5,66 \pm 2,12$ & $\mathrm{P}<0,05$ \\
Posição perineal na evacuação & $9,21 \pm 2,02$ & $7,61 \pm 1,90$ & $\mathrm{P}<0,05$ \\
\hline
\end{tabular}


ABSTRACT: INTRODUCTION - Rectocele is a common cause of constipation for obstructed defecation. It often is related with other causes of outlet obstruction and the examinations of anal physiology tests are important for necessary diagnosis of the constipation cause. OBJECTIVE - To observe the influence of rectocele in the anal physiology tests and the necessity of its accomplishment to the diagnosis of rectocele. METHOD In this study, 40 patients with diagnosis of outlet obstruction had been analyzed. All the patients had been submitted to manometry, rectal sensitivity, electromyography, pudendal nerve latency and proctography and they had been separated in 2 groups: carriers and non-carriers of rectocele. 18 patients with rectocele had been found and the diagnosis often associated was anismus. In relation to the examination there was significant difference only in the proctography in the rectal angle, demonstrating that the presence of rectocele did not intervene with the results of the examination of anal physiology when compared with the other constipated, but it was associated with other causes of constipation such as anismus that needs another type of treatment. CONCLUSÃO - Rectocele did not intervene with the values of the examination of anal physiology, but it was associated with other diagnosis, therefore it is important the accomplishment of the examination.

Key words: Rectocele; Constipation; Physiological Examinations.

\section{REFERÊNCIAS}

1. AGUIDA, H.A.C. - Efeito da idade e do sexo nas pressões do canal anal - estudo manométrico. São Paulo, 1996. (Tese — Mestrado - Faculdade de Ciências Médicas da Santa Casa de São Paulo).

2. BLEIJENBERG, G.; KUIJPERS, H.C. - Biofeedback treatment of constipation: a comparison of two methods. AJG, 89(7):1021-6, 1994.

3. CAMILLERI, M.; GRANT THOMPSON, W.; FLESHMAN, J.W.; PEMBERTON, J.H. - Clinical management of intractable constipation. Ann. Intern. Med., 121(7):520-8, 1994.

4. HALligAN, S. \& BARTRAN, C.I. - Evacuation proctography combined with positive contrast peritoneography to demonstrate pelvic floor hernias. Abdom. Imaging., 20(5):442-5, 1995.

5. HILTUNEN, K.M.; KOLEHMAINEN, H.; MATIKAINEN, M. - Does defecography help in diagnosis and clinical decision-making in defecation disorders? Abdom. Imaging., 19:355-8, 1994

6. KOUTSOMANIS, D.; LENNARD-JONES, J.E.; ROY, A.J.; KAMM, M.A. - Controlled randomised trial of visual biofeedback versus muscle training without a visual display for intractable constipation. GUT, 37:95-9, 1995.

7. MELLGREN, A.; BREMMER, S.; JOHANSSON, C.; DOLK, A.; UDÉN, R.; AHLBACK, S-O.; HOLMSTRON, B. - Results of rectocele repair - a prospective study. Dis. Colon. Rectum., 37:1133-41, 1994.
8. MELLGREN, A. - Diagnosis and treatment of constipation. Eur. J. Surg., 161:623-34, 1995.

9. MELlGREN, A.; ANZEN, B.; NILSSON, B.Y.; JOHANSSON, C.; DOLK, A.; GILLGREN, P.; BREMMER, S.; HOLMSTRO, B. - Results of rectocele repair - a prospective study. Dis. Colon. Rectum, 38(1):7-13, 1995.

10. OTT, D.J.; DONATTI, D.L.; KERR, R.M.; CHEN, M.Y.M. - Defecography: results in 55 patients and impact on clinical management. Dis. Colon. Rectum., 40(3):280-6, 1997.

11. PFEIFER, J.; TEOH, T-A.; SALANGA, V.D.; AGACHAN, V.F.; WEXNER, S.D. - Comparative study between intraanal sponge and needle electrode for electromyographic evaluation of constipated patients. Dis. Colon. Rectum., 41:1153-7, 1998.

12. SCHOUTEN, W.R.; BRIEL, J.W.; AUERDA, J.J.A.; VANDAM, J.H.; GOSSELINK, M.J.; GINAI, A.Z.; HOP, W.C.J. - Anismus: fact or fiction? Dis. Colon. Rectum., 40:1033-41, 1997.

13. VACCARO, C.A.; CHEONG, D.M.O.; WEXNER, S.D.; NOGUERAS, J.J.; SALANGA, V.D.; HANSON, M.R.; PHILLIPS, R.C. - Pudendal neuropathy in evacuatory disorders. Dis. Colon. Rectum., 38:166-71, 1995.

Endereço para correspondência: MARIA AUXILIADORA PROLUNGATTI CESAR RUA VINICIUS DE MORAES , 434 - CAMPOS DO CONDE I TREMEMBÉ -SP 